\title{
AMS-02 antiprotons' consistency with a secondary astrophysical origin
}

\author{
Mathieu Boudaud,,$^{1, *}$ Yoann Génolini $\odot,{ }^{2, \dagger}$ Laurent Derome $\odot,{ }^{3}$ Julien Lavalle $\odot,{ }^{4}$ David Maurin $\odot,{ }^{3}$ \\ Pierre Salati $\odot,{ }^{5}$ and Pasquale D. Serpico $\odot^{5}$ \\ ${ }^{1}$ LPTHE, Sorbonne Université, CNRS, 4 Place Jussieu, F-75005 Paris, France \\ ${ }^{2}$ Service de Physique Théorique, Université Libre de Bruxelles, Boulevard du Triomphe, CP225, 1050 Brussels, Belgium \\ ${ }^{3}$ LPSC, Université Grenoble Alpes, CNRS, 53 avenue des Martyrs, F-38000 Grenoble, France \\ ${ }^{4}$ LUPM, Université de Montpellier, CNRS, Place Eugène Bataillon, F-34000 Montpellier, France \\ ${ }^{5}$ LAPTh, Université Grenoble Alpes, USMB, CNRS, F-74000 Annecy, France
}

(Received 19 June 2019; revised manuscript received 12 September 2019; accepted 27 February 2020; published 9 April 2020)

\begin{abstract}
The Alpha Magnetic Spectrometer (AMS-02) experiment has ushered cosmic-ray physics into precision era. In an earlier paper [Génolini et al., Phys. Rev. D 99, 123028 (2019)], we designed an improved method to calibrate propagation models on the boron-to-carbon ratio data. Here we provide a robust prediction of the $\bar{p}$ flux, accounting for several sources of uncertainties and their correlations. Combined with a correlation matrix for the $\bar{p}$ data, we show that the latter are consistent with a secondary origin. This paper presents key elements relevant to the dark matter search in this channel, notably by pointing out the inherent difficulties in achieving predictions at the percent-level precision.
\end{abstract}

DOI: 10.1103/PhysRevResearch.2.023022

\section{INTRODUCTION}

The spectrum of Galactic cosmic-ray (CR) antiprotons $(\bar{p}$ 's) originating from interactions of $\mathrm{CR}$ protons on the interstellar (IS) gas was first calculated fifty years ago [1,2]. CR $\bar{p}$ 's were discovered a few years later in balloon-borne experiments [3,4], but in excess compared to this expected secondary background; this was soon interpreted as the presence of an extra contribution, possibly from primordial black holes [5] or dark matter (DM) annihilation [6,7]. Over the past thirty years, a lot of progress has been made both theoretically and experimentally.

From the 1990s to the 2000s, balloon flights (MASS, IMAX, CAPRICE, HEAT-pbar, and BESS [8-17]) and AMS01 onboard the shuttle [18] collected hundreds of $\bar{p}$ 's, up to $\approx 10 \mathrm{GeV}$. Improvements in the predictions, accounting for tertiary production [19], more accurate cross sections [20], and reacceleration [21] paved the road to obtain compatibility of the data with modern diffusion models [22,23], the transport parameters of which were fitted on the boron-to-carbon ratio $(\mathrm{B} / \mathrm{C})[24]$.

In the following decade, the next generation of instruments-BESS-TeV/Polar [25-27] and the PAMELA satellite [28-30]-measured several thousands of $\vec{p}$ 's up to a few hundreds of $\mathrm{GeV}$. Updates of the model parameters and

\footnotetext{
${ }^{*}$ Deceased.

†yoann.genolini@ulb.ac.be

Published by the American Physical Society under the terms of the Creative Commons Attribution 4.0 International license. Further distribution of this work must maintain attribution to the author(s) and the published article's title, journal citation, and DOI.
}

corresponding predictions, using better measured $p$ and $\mathrm{He}$ CR spectra, yielded a secondary flux in agreement with the data (e.g., Ref. [31]).

The state-of-the-art AMS-02 experiment has been operating on the International Space Station (ISS) since 2011. It has already recorded several tens of thousands of $\bar{p}$ 's [32], up to $\mathrm{TeV}$ energies. For the first time, the measurement is dominated by systematic uncertainties (at a few percent level), implying new challenges for their interpretation. Here we address this issue, going beyond previous analyses of preliminary AMS-02 $\bar{p}$ 's data [33-35].

We underline that CR $\vec{p}$ 's are one of the most sensitive astroparticle probes of annihilating and decaying DM in the $\mathrm{GeV}-\mathrm{TeV}$ range [36-40], and any constraint on DM candidates depends on how well the astrophysical background is controlled. This is especially important as claims for $\bar{p}$ excesses attributed to DM are being debated [41-45].

\section{METHODOLOGY}

The flux of CR $\bar{p}$ 's at Earth depends on (i) the cross sections entering their production, scattering, and annihilation, (ii) the CR propagation model, (iii) the IS spectrum of the (most abundant) CR nuclei, and (iv) modulation of fluxes in the Solar cavity.

Uncertainties on relevant nuclear cross sections are among the dominant ones for the $\bar{p}$ flux calculation [22,33]. Recently, new data have been taken [46-48], leading to improvements in the cross section parametrizations [49-53]. A crucial ingredient needed for the calculation is the Lorentz-invariant cross section of prompt $\bar{p}$ 's produced in $p p$ interactions. We make use of the parametrization proposed in Ref. [52] and improved in Ref. [53] (Param II). For nucleon-nucleon interactions, we use the scaling relation B proposed in Ref. [53], which 
provides the best agreement with Large Hadron Collider beauty data. We use the covariance matrices of errors on the parameters to propagate the uncertainties to the $\bar{p}$ flux calculation. Antineutrons ( $\bar{n}$ 's) and antihyperons are produced in hadronic interactions and decay into $\bar{p}$ 's; their contribution has to be included in the total cross section. The yield of $\bar{p}$ 's produced via $\bar{n}$ 's is larger than in the prompt $\bar{p}$ channel, an energy-dependent effect studied and parameterised in Ref. [52], in agreement with the very scarce experimental data. We introduce an exponentially decreasing energydependent parametrization in order to reproduce the $1 \sigma$ confidence level (CL) interval of Fig. 8 in Ref. [52]. Regarding the antihyperon contribution, we make use of the parametrization determined in Ref. [52] and the covariance matrix of the corresponding parameters to propagate the uncertainties on the $\bar{p}$ flux calculation. More details on the nonprompt channels and on the way we propagate the related uncertainties are given in the Supplemental Material (SM [54]), Secs. I-A and $\mathrm{I}-\mathrm{B}$, respectively. Inelastic annihilating and nonannihilating interactions of $\bar{p}$ 's with the IS medium (ISM) are treated following the procedure described in Ref. [38].

We use the one-dimensional (1D) diffusion model implemented in USINE V3.5 [55], which assumes a thick diffusion halo size $L$ and a thin disk containing the sources and the gas. Computationally more expensive scenarios with extended geometries [50,56,57] and time dependence [58] have been considered in the literature. Yet, should simple 1D models prove sufficient to account for the data in a secondary production scenario, more complex scenarios would a fortiori work as well. We use the most generic transport model defined in Ref. [59] (called BIG), where the transport parameters are fitted on $\mathrm{B} / \mathrm{C}$ following the methodology described in Ref. [60]; i.e., a model of the covariance matrix of $\mathrm{B} / \mathrm{C}$ AMS-02 errors has also been incorporated in the fit. Using the MINUIT [61] package to fit the B/C, we compute the best-fit values of the free parameters (transport and nuisance parameters) and their covariance matrix. We fully propagate the transport uncertainty to the $\bar{p}$ flux from the covariance matrix. We have compared the distribution of the best-fit parameters from the covariance matrix with the distribution obtained by resampling the $\mathrm{B} / \mathrm{C}$ data to check that the Gaussian treatment of the uncertainties is valid in this context.

Important inputs for the $\bar{p}$ calculation are the IS fluxes for all progenitors [mostly $\mathrm{H}, \mathrm{He}, \mathrm{C}$, and $\mathrm{O}$, but also all nuclei up to $\mathrm{Fe}$ (see SM Sec. II-C [54] for their detailed contributions); we also account for heavy elements in the ISM (see SM II-D)]. There are two options to propagate the associated uncertainties: a standard approach in semianalytical models $[22,31,33,52]$ is to demodulate top-of-atmosphere (TOA) data, in order to extract the parameters describing the IS fluxes and their uncertainties, which allows us to yield the $\bar{p}$ flux with a fast procedure and to easily propagate the progenitors uncertainties. The preferred approach in fully numerical propagation codes is to directly use the IS fluxes calculated at the propagation stage, ensuring that the calculated fluxes fit the data $[23,35,56,62]$. In this global fitting, the transport and source uncertainties are determined simultaneously. Although using a semianalytical model, we follow this second approach but do not rely on a global fitting of the transport and source parameters. Instead, we follow and extend the two-step procedure detailed in Ref. [59] (see Sec. III therein): We start from the best-fit parameters obtained from the $\mathrm{B} / \mathrm{C}$ analysis (BIG) and then perform a simultaneous fit of $\mathrm{H}$ [63], $\mathrm{He}, \mathrm{C}$, and $\mathrm{O}$ [64] AMS-02 data to determine the source parameters, i.e., four normalizations $\left({ }^{1} \mathrm{H},{ }^{4} \mathrm{He},{ }^{12} \mathrm{C}\right.$, and $\left.{ }^{16} \mathrm{O}\right)$ and three slopes $\left(\alpha_{\mathrm{H}}, \alpha_{\mathrm{He}}\right.$, and a universal source slope $\alpha_{\mathrm{Z}>2}$ for all other species). Source isotopic fractions are fixed to Solar System values [65]; the abundances of nonfitted elements up to $Z=30$ are fixed such as to match the HEAO-3 data [66] at $10.6 \mathrm{GeV} / \mathrm{n}$. We fit at the same time the high-rigidity diffusion break parameters which are better constrained by elemental fluxes than by the B/C [59]. We include in the fit the covariance matrices of errors on $\mathrm{H}, \mathrm{He}, \mathrm{C}$, and $\mathrm{O}$ data; see SM Sec. II-A [54]. The outputs of this fitting procedure are discussed in SM Sec. II-B. Note that we fix the Fisk potential ${ }^{1} \phi_{\mathrm{FF}}$ for $\mathrm{H}, \mathrm{He}, \mathrm{C}$, and $\mathrm{O}$ data to the value yielded by the $\mathrm{B} / \mathrm{C}$ data fit [59]. While published AMS-02 data are from the same time period for B/C, He, C, and O (05/2011 to 05/2016), they originate from a shorter period for $\mathrm{H}(05 / 2011$ to $11 / 2013)$, so that the associated modulation level should be slightly different. However, as the fit is restricted to data above the $\bar{p}$ production threshold $\left(E_{k / n}>6 m_{p}[22]\right)$, the impact is feeble, and in any case negligible compared to other uncertainties.

State-of-the-art modulation models are 2D or 3D chargedependent diffusion and drift in the Solar cavity [72]. They go beyond the force-field approximation [73] and, at variance with the latter, they predict different modulations for positively and negatively charged particles. Charge-signdependent effects on secondary antiprotons have been estimated by Ref. [74] to be smaller than $\approx 10 \%$ below $5 \mathrm{GeV}$ and negligible above. However, these models have a large number of parameters and are still under study. Moreover, the impact of the drift effect is strongly dependent on the data-collection period. The AMS-02 $\bar{p}$ data [32] were analyzed from a time period of 4 years (May 2011 to May 2015) longer than that of $\mathrm{H}$ and shorter than that of $\mathrm{He}, \mathrm{C}$, and $\mathrm{O}$ data. As illustrated in Ref. [75], drift models can be used to derive effective and different Fisk potentials for nuclei and $\bar{p}$ 's, with $\phi_{\mathrm{FF}}^{\bar{p}}$ which is not clearly smaller or larger than $\phi_{\mathrm{FF}}^{\text {nuc }}$ on a sufficiently long period. Assuming the same modulation for $\bar{p}$ and $\mathrm{H}$, $\mathrm{He}, \mathrm{C}$, and $\mathrm{O}$ actually already gives a satisfactory description at low rigidity. For this reason, we did not include further uncertainty on $\phi_{\mathrm{FF}}^{\bar{p}}$. If anything, this would slightly enlarge the uncertainty of the prediction at low rigidity, improving further the consistency between our calculated $\bar{p}$ flux and the data.

Uncertainties in the parameters discussed above should be propagated to the $\bar{p}$ flux calculation. To proceed, for each source of uncertainty (production cross section, transport in the Galaxy, fit to parents fluxes) we draw $\approx 10000$ realizations of the parameters from their covariance matrix and we compute the corresponding $\bar{p}$ fluxes. We then calculate the $1 \sigma$ confidence-level (CL) envelope for each source of uncertainty as well as the covariance matrix of the model $\mathcal{C}^{\text {model }}$ (see SM Sec. III [54]). This enables us to soundly assess the compatibility of the model with the data.

\footnotetext{
${ }^{1}$ The force-field approximation [67-70] has a single free parameter, and it was found to describe well-modulated fluxes [71].
} 


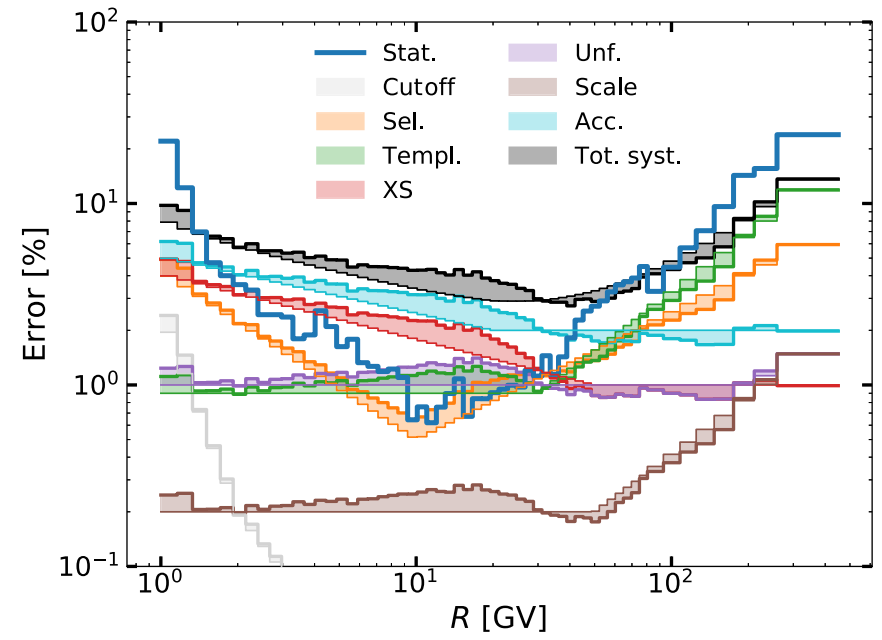

FIG. 1. AMS-02 errors for $\bar{p}$ data. Statistical (Stat.) and total systematic (Tot. syst.) lines correspond to the errors provided in Ref. [76]. Individual contributions in the systematic errors, namely rigidity cutoff (Cutoff), selection (Sel.), template fitting (Templ.), cross sections (XS), unfolding (Unf.), rigidity scale (Scale), and acceptance (Acc.), built from information provided in Ref. [32] are shown (coloured lines) before (thin) and after (thick) the rescaling applied to match the total systematic error.

In the context of the $\mathrm{B} / \mathrm{C}$ analysis, we stressed the importance of using a realistic covariance matrix of the data errors to avoid misleading conclusions [60]. We anticipate that the same is true for $\bar{p}$ 's. However, since this matrix is not directly provided by the AMS- 02 collaboration, we build it from the published systematic errors and associated description of their physics origin, in the same spirit as in Ref. [60].

The various contributions to the AMS-02 systematics are broadly described in Ref. [32]. For instance page 5, the text "[t]his [selection] uncertainty amounts to $4 \%$ at $1 \mathrm{GV}, 0.5 \%$ at $10 \mathrm{GV}$, and rises to $6 \%$ at $450 \mathrm{GV}$ " is interpreted as a piecewise power-law behavior, and is shown as an orange thin line in Fig. 1. Thus, we build the seven sources of systematics quoted by the AMS-02 collaboration (colored thin lines), where the quadratic sum of all contributions leads to the black thin line. In order for the sum to match the total systematic errors provided in Ref. [32] (black thick line), we rescale for each rigidity point our separate contributions by the ratio of the thick to the thin black lines. This leads to our model for the AMS-02 $\bar{p}$ systematics (colored thick lines). The covariance matrix associated with these systematics is then built based on a choice of their correlation length, $\ell$. More details on this procedure are given in SM Sec. III [54] and, for the B/C analysis, in Ref. [60]. For $\bar{p}$ 's, we take as educated guesses for the correlations lengths (in unit of energy decade) $\ell_{\text {Acc. }}=0.1$ (acceptance), $\ell_{\text {Cut }}=1.0$ (rigidity cutoff), $\ell_{\text {scale }}=4.0$ (rigidity scale), $\ell_{\text {Templ. }}=0.5$ (template fitting), $\ell_{\mathrm{XS}}=1.0$ (cross sections), $\ell_{\text {Unf. }}=1.0$ (unfolding), and $\ell_{\text {Sel. }}=0.5$ (selection).

\section{RESULTS}

The top panel of Fig. 2 shows our baseline $\bar{p}$ flux prediction (not a fit) obtained from the best-fit values for the $\bar{p}$ production

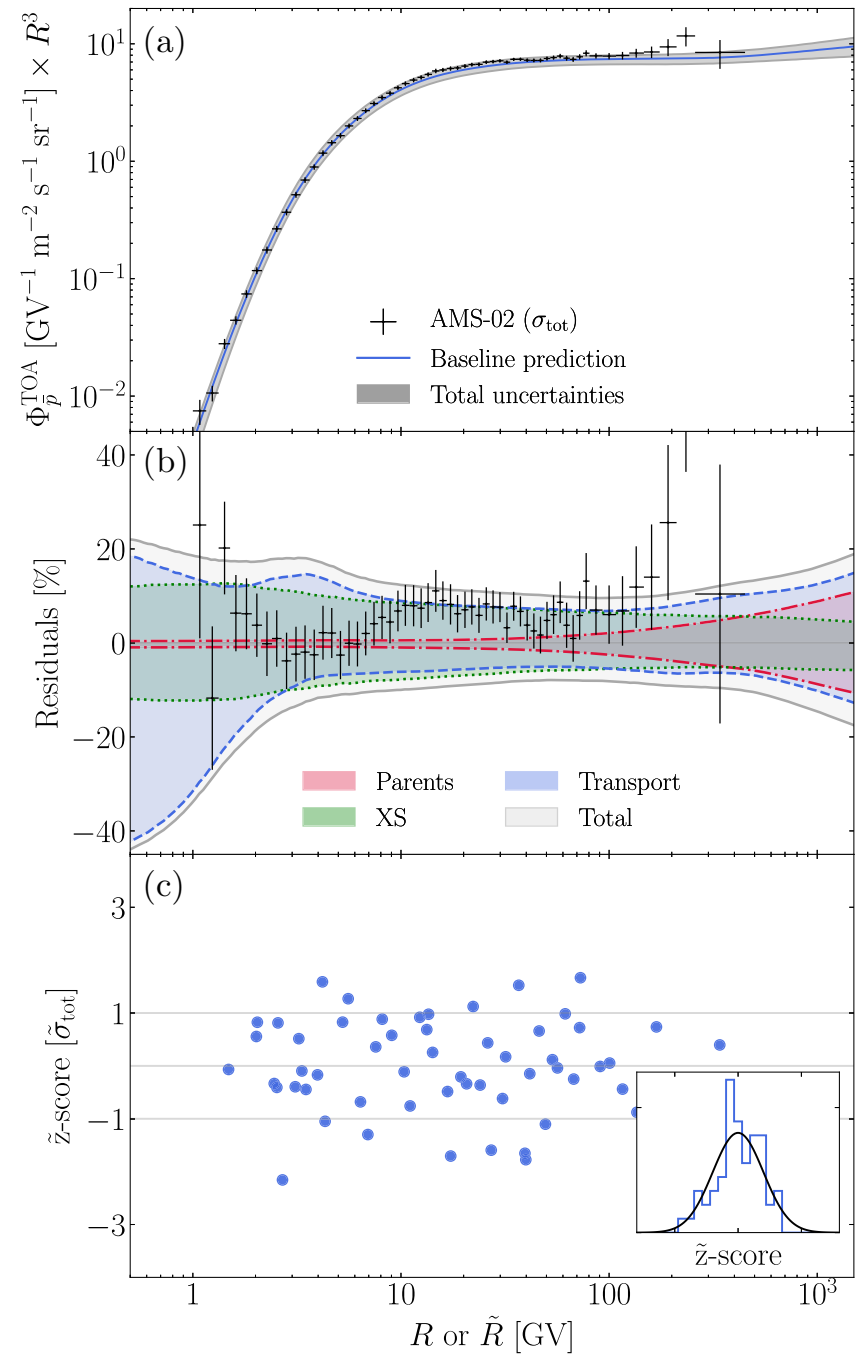

FIG. 2. Comparison of $\bar{p}$ model and data (a), along with residuals and $68 \%$ total confidence interval for the model (gray) together with the transport (blue), the parents (red), and the cross section (green) contributions (b). The residuals of the eigenvectors of the total covariance matrix as a function of the pseudorigidity $\tilde{R}$, as well as their distribution are shown in panel (c) and in the inset.

cross sections, the transport (BIG), and the associated parents' fluxes, compared with AMS-02 data with errors taken as the quadratic sum of systematic and statistical errors (black crosses). The "standard" residuals with respect to the baseline model are displayed in the middle panel. Note that the points do not include the model uncertainties, nor correlations in the data uncertainties. We also show on the same plot the $68 \%$ total confidence band for the model (gray band). This band could release the tension with the data, even before accounting for the information on the correlations in rigidity bins. The respective contributions of parents, cross sections, and transport are also plotted. At tens of GV, the errors from transport and cross sections are almost constant and close to $10 \%$. At larger rigidities, the errors from transport and parents increase because of the increasing experimental uncertainty in the $\mathrm{B} / \mathrm{C}$ ratio and parent fluxes, respectively. At low rigidity, the error from transport grows for the same reasons and encompasses the uncertainty in the prediction of 
TABLE I. Respective $p$ values for different sources of errors. We take dof $=57$, i.e., the number of $\bar{p}$ data. Total errors on data are defined to be $\sigma_{\text {tot }}=\sqrt{\sigma_{\text {stat }}^{2}+\sigma_{\text {syst }}^{2}}$.

\begin{tabular}{lccc}
\hline \hline Error considered & $\chi^{2} /$ dof & $p$ value $\left(\chi^{2}\right)$ & $p$ value $(\mathrm{KS})$ \\
\hline$\sigma_{\text {stat }}$ & 23 & 0 & 0 \\
$\sigma_{\text {tot }}$ & 1.69 & $8.3 \times 10^{-4}$ & 0 \\
$\mathcal{C}^{\text {data }}$ & 0.85 & 0.79 & 0.97 \\
$\sigma_{\text {stat }}$ and $\mathcal{C}^{\text {model }}$ & 1.32 & 0.05 & 0.99 \\
$\sigma_{\text {tot }}$ and $\mathcal{C}^{\text {model }}$ & 0.37 & 1.0 & 0.01 \\
$\mathcal{C}^{\text {data }}$ and $\mathcal{C}^{\text {model }}$ & 0.77 & 0.90 & 0.86 \\
\hline \hline
\end{tabular}

the low-rigidity behavior (see SM Sec. V [54]). However, we remind the reader that a visual comparison can be deceiving: The presence of nondiagonal values in the covariance matrices is responsible for a better agreement between the model and data than perceived in the residuals (see Table I).

To test the actual compatibility of our prediction with the $\bar{p}$ data, we present two statistical tests which boil down to probabilistic statements in terms of $p$ value (see SM Sec. IV [54]). First, we propose a $\chi^{2}$ test, with the help of a covariance matrix of errors on both data and model:

$\chi^{2}=(\text { data }- \text { model })^{\mathrm{T}}\left(\mathcal{C}^{\text {model }}+\mathcal{C}^{\text {data }}\right)^{-1}($ data - model $)$.

The covariance matrices of the data $\mathcal{C}^{\text {data }}$ and the model $\mathcal{C}^{\text {model }}$ are given by the sum of the different contributions previously detailed (see SM, Secs. II-A and III [54]). We find $\chi^{2} \approx 44$, and by identifying the number of degrees of freedom (dof) with the number of $\bar{p}$ data points (57), we infer a corresponding $p$ value of 0.9 , which is reported in the last line of Table I.

Such a test does not directly assess a possible overestimate of the errors and also relies on the notion of number of degrees of freedom (dof,which may be a shaky concept in some circumstances; see, e.g., the discussion in Ref. [77]). Thus, we also perform a Kolmogorov-Smirnov (KS) test, which obviates the above limitations. We compute the distribution of the "eigenresiduals" ( $\tilde{z}$ score $)$ corresponding to the residuals of the eigenvectors (data model) of the total covariance matrix. In the bottom panel of Fig. 2 we show these eigenresiduals as a function of rigidity (actually, the one rotated in the eigenbasis; see SM IV [54]), and in the inset, we compare the corresponding histogram with a Gaussian. The KS test leads to a $p$ value of 0.27 , which is also very good and is clearly consistent with the hypothesis that $\bar{p}$ 's are of secondary origin. For completeness, we also report in Table I the $p$ values when considering different combinations of errors: (i) If there was no uncertainty in our baseline model, the covariance matrix of data errors alone $\left(\mathcal{C}^{\text {data }}\right)$ would already give enough freedom to allow for a very good agreement between the data and the secondary flux prediction and (ii) considering only the statistical uncertainties in the data and the uncertainties in the model $\left(\sigma_{\text {stat }}\right.$ and $\left.\mathcal{C}^{\text {model }}\right)$, this prediction is marginally consistent with the data at the $2 \sigma$ level, with the KS test leading to an even better $p$ value. Also note the relevance of the KS test (as opposed to the $\chi^{2}$ test) to spot error overestimates, in the case of $\sigma_{\text {tot }}$ and $\mathcal{C}^{\text {model }}$; (iii) In the most realistic case considering both $\mathcal{C}^{\text {data }}$ and $\mathcal{C}^{\text {model }}, p$-values are very good for both the $\chi^{2}$ and KS test. Thus, not only is a secondary origin for the locally measured $\bar{p}$ 's statistically consistent with the data, but, as shown by these considerations, it is also robust with respect to error mismodeling in either model or data errors.

\section{CONCLUSIONS}

Percent-level details in the model predictions now matter, as do more subtle aspects of the data error treatment. In this paper, we have presented a major upgrade of the $\bar{p}$ flux prediction and analysis by (i) using the latest constraints on transport parameters from AMS-02 B/C data, (ii) propagating all uncertainties (with their correlations) on the predicted $\bar{p}$ flux, and (iii) accounting for correlated errors in $\bar{p}$ data. The multicomponent nature of the systematic error, with different $R$ dependencies and correlation lengths, has a crucial impact on the analysis and was not captured in more simplified treatments as in Ref. [44]. With these novelties, we unambiguously show that the AMS-02 data are consistent with a pure secondary astrophysical origin. We stress that this conclusion is not based on a fit to the AMS-02 $\bar{p}$ data but on a prediction of the $\bar{p}$ flux computed from external data. Our results should hold for any steady-stade propagation model of similar complexity, as they all amount to the same "effective grammage" crossed to produce boron nuclei (on which the analysis is calibrated), with roughly the same grammage entering the secondary $\bar{p}$ 's. We have checked that this conclusion is robust with respect to a variation by a factor of a few of the correlation lengths of the AMS-02 systematic uncertainties. Also, recent analyses of Fermi-LAT data are suggestive of a spatial dependent diffusion coefficient, notably different in the inner Galaxy [78]. Moving to more complex scenarios containing the 1D framework considered here as limiting case would broaden theory space but would not alter our conclusions on the viability of secondary production to explain antiproton data. On the technical aspects, more computationally expensive methods could allow one to go beyond the quadratic assumption (i.e., assuming multi-Gaussian error distributions) embedded in the covariance matrix of errors. For more advanced applications, sampling techniques like Markov chain Monte Carlo could be used (e.g., Ref. [79]). However, a significant improvement in our perspectives for DM searches in the $\bar{p}$ flux can only be achieved by simultaneously reducing the systematics in the data and the errors of the modeling. On the data side, a covariance matrix of errors directly provided by the AMS- 02 Collaboration would definitively be an important improvement to fully benefit from the precision achieved by AMS-02. On the modeling side, the next step would be to combine more secondary-to-primary ratios $(\mathrm{Li} / \mathrm{C}, \mathrm{Be} / \mathrm{C}$, and $\mathrm{B} / \mathrm{C})$ to further decrease the propagation uncertainties. Of course, better data and modeling on $\bar{p}$ and $\bar{n}$ production cross sections is also required, and the subleading error due to primary source parameters could be reduced by combining AMS-02 data with higher energy data from CREAM, TRACER, and CALET [80]. In the current state of our analysis, we can anticipate that, from the frequentist point of view, a clear statistical preference for an additional feature in the data is unlikely. However, this conclusion must rely on a quantitative analysis that we postpone for a forthcoming paper. 


\section{ACKNOWLEDGMENTS}

M.B. is grateful to Michael Korsmeier and Martin Winkler for very useful discussions. We are grateful to all the members of the Cosmic Rays Alpine Collaboration. This work has been supported by the "Investissements d'avenir, Labex ENIGMASS," by Univ. de Savoie AAP "DISE," and by the French ANR, Project DMAstro-LHC, ANR-12-BS05-0006. The work of Y.G. is supported by the IISN, the FNRS-FRS, and a ULB ARC. We also acknowledge partial support from the Agence Nationale pour la Recherche (ANR) Project No. ANR-18-CE31-0006, the Origines, Constituants, et EVolution de l'Univers (OCEVU) Labex (No. ANR-11-LABX0060), the CNRS IN2P3-Theory/INSU-PNHE-PNCG project "Galactic Dark Matter," and the European Union's Horizon 2020 research and innovation program under the Marie Sklodowska-Curie Grant Agreements No. 690575 and No. 674896.
[1] S. Rosen, Phys. Rev. 158, 1227 (1967).

[2] C. S. Shen and G. B. Berkey, Phys. Rev. 171, 1344 (1968).

[3] E. A. Bogomolov, N. D. Lubianaia, V. A. Romanov, S. V. Stepanov, and M. S. Shulakova, International Cosmic Ray Conference 9, 146 (1981).

[4] A. Buffington, S. M. Schindler, and C. R. Pennypacker, Ap. J. 248, 1179 (1981).

[5] P. Kiraly, J. Szabelski, J. Wdowczyk, and A. W. Wolfendale, Nature (London) 293, 120 (1981).

[6] J. Silk and M. Srednicki, Phys. Rev. Lett. 53, 624 (1984).

[7] F. W. Stecker, S. Rudaz, and T. F. Walsh, Phys. Rev. Lett. 55, 2622 (1985).

[8] M. Hof, W. Menn, C. Pfeifer, M. Simon, R. L. Golden, S. J. Stochaj, S. A. Stephens, G. Basini, M. Ricci, F. M. Brancaccio et al., Ap. J. 467, L33 (1996).

[9] J. W. Mitchell, L. M. Barbier, E. R. Christian, J. F. Krizmanic, K. Krombel, J. F. Ormes, R. E. Streitmatter, A. W. Labrador, A. J. Davis, R. A. Mewaldt et al., Phys. Rev. Lett. 76, 3057 (1996).

[10] M. Boezio, P. Carlson, T. Francke, N. Weber, M. Suffert, M. Hof, W. Menn, M. Simon, S. A. Stephens, R. Bellotti et al., Ap. J. 487, 415 (1997).

[11] M. Boezio, V. Bonvicini, P. Schiavon, A. Vacchi, N. Zampa, D. Bergström, P. Carlson, T. Francke, S. Grinstein, M. Suffert et al., Ap. J. 561, 787 (2001).

[12] A. S. Beach, J. J. Beatty, A. Bhattacharyya, C. Bower, S. Coutu, M. A. Duvernois, A. W. Labrador, S. McKee, S. A. Minnick, D. Müller, J. Musser, S. Nutter, M. Schubnell, S. Swordy, G. Tarlé, and A. Tomasch, Phys. Rev. Lett. 87, 271101 (2001).

[13] A. Moiseev, K. Yoshimura, I. Ueda, K. Anraku, R. Golden, M. Imori, S. Inaba, B. Kimball, N. Kimura, Y. Makida et al. (BESS Collaboration), Ap. J. 474, 479 (1997).

[14] H. Matsunaga, S. Orito, H. Matsumoto, K. Yoshimura, A. Moiseev, K. Anraku, R. Golden, M. Imori, Y. Makida, J. Mitchell et al., Phys. Rev. Lett. 81, 4052 (1998).

[15] S. Orito, T. Maeno, H. Matsunaga, K. Abe, K. Anraku, Y. Asaoka, M. Fujikawa, M. Imori, M. Ishino, Y. Makida et al., Phys. Rev. Lett. 84, 1078 (2000).

[16] T. Maeno, S. Orito, H. Matsunaga, K. Abe, K. Anraku, Y. Asaoka, M. Fujikawa, M. Imori, Y. Makida, N. Matsui et al., Astropart. Phys. 16, 121 (2001).

[17] Y. Asaoka, Y. Shikaze, K. Abe et al., Phys. Rev. Lett. 88, 051101 (2002).

[18] M. Aguilar, J. Alcaraz, J. Allaby, B. Alpat, G. Ambrosi, H. Anderhub, L. Ao, A. Arefiev, P. Azzarello et al. (AMS Collaboration), Phys. Rep. 366, 331 (2002).
[19] L. C. Tan and L. K. Ng, J. Phys. G: Nucl. Part. Phys. 9, 227 (1983).

[20] T. K. Gaisser and R. K. Schaefer, Ap. J. 394, 174 (1992).

[21] M. Simon, A. Molnar, and S. Roesler, Ap. J. 499, 250 (1998).

[22] F. Donato, D. Maurin, P. Salati, A. Barrau, G. Boudoul, and R. Taillet, Ap. J. 563, 172 (2001).

[23] I. V. Moskalenko, A. W. Strong, J. F. Ormes, and M. S. Potgieter, Ap. J. 565, 280 (2002).

[24] D. Maurin, F. Donato, R. Taillet, and P. Salati, Ap. J. 555, 585 (2001).

[25] S. Haino, K. Abe, H. Fuke, T. Maeno, Y. Makida, H Matsumoto, J. W. Mitchell, A. A. Moiseev, J. Nishimura, M. Nozaki et al., in Proceedings of the 29th International Cosmic Ray Conference Pune (2005), Vol. 3, p. 13.

[26] K. Abe, H. Fuke, S. Haino, T. Hams, A. Itazaki, K. C. Kim, T. Kumazawa, M. H. Lee, Y. Makida, S. Matsuda, K. Matsumoto et al. (BESS Collaboration), Phys. Lett. B 670, 103 (2008).

[27] K. Abe, H. Fuke, S. Haino, T. Hams, M. Hasegawa, A. Horikoshi, K. C. Kim, A. Kusumoto, M. H. Lee, Y. Makida et al., Phys. Rev. Lett. 108, 051102 (2012).

[28] O. Adriani, G. C. Barbarino, G. A. Bazilevskaya, R. Bellotti, M. Boezio, E. A. Bogomolov, L. Bonechi, M. Bongi, V. Bonvicini, S. Bottai et al., Phys. Rev. Lett. 102, 051101 (2009).

[29] O. Adriani, G. C. Barbarino, G. A. Bazilevskaya, R. Bellotti, M. Boezio, E. A. Bogomolov, L. Bonechi, M. Bongi, V. Bonvicini, and S. Borisov, Phys. Rev. Lett. 105, 121101 (2010).

[30] O. Adriani, G. A. Bazilevskaya, G. C. Barbarino, R. Bellotti, M. Boezio, E. A. Bogomolov, V. Bonvicini, M. Bongi, L. Bonechi et al., JETP Lett. 96, 621 (2013).

[31] F. Donato, D. Maurin, P. Brun, T. Delahaye, and P. Salati, Phys. Rev. Lett. 102, 071301 (2009).

[32] M. Aguilar, L. Ali Cavasonza, B. Alpat, G. Ambrosi, L. Arruda, N. Attig, S. Aupetit, P. Azzarello, A. Bachlechner, F. Barao et al. (AMS Collaboration), Phys. Rev. Lett. 117, 091103 (2016).

[33] G. Giesen, M. Boudaud, Y. Génolini, V. Poulin, M. Cirelli, P. Salati, and P. D. Serpico, J. Cosmol. Astropart. Phys. 09 (2015) 023.

[34] R. Kappl, A. Reinert, and M. W. Winkler, J. Cosmol. Astropart. Phys. 10 (2015) 034.

[35] C. Evoli, D. Gaggero, and D. Grasso, J. Cosmol. Astropart. Phys. 12 (2015) 039.

[36] D. Maurin, R. Taillet, F. Donato, P. Salati, A. Barrau, and G. Boudoul, arXiv:astro-ph/0212111.

[37] J. Lavalle and P. Salati, C.R. Phys. 13, 740 (2012). 
[38] M. Boudaud, M. Cirelli, G. Giesen, and P. Salati, J. Cosmol. Astropart. Phys. 05 (2015) 013.

[39] J. Conrad and O. Reimer, Nat. Phys. 13, 224 (2017).

[40] S. J. Clark, B. Dutta, and L. E. Strigari, Phys. Rev. D 97, 023003 (2018).

[41] M.-Y. Cui, Q. Yuan, Y.-L. S. Tsai, and Y.-Z. Fan, Phys. Rev. Lett. 118, 191101 (2017).

[42] A. Cuoco, M. Krämer, and M. Korsmeier, Phys. Rev. Lett. 118, 191102 (2017).

[43] I. Cholis, T. Linden, and D. Hooper, Phys. Rev. D 99, 103026 (2019).

[44] A. Cuoco, J. Heisig, L. Klamt, M. Korsmeier, and M. Krämer, Phys. Rev. D 99, 103014 (2019).

[45] S.-J. Lin, X.-J. Bi, and P.-F. Yin, Phys. Rev. D 100, 103014 (2019).

[46] T. Anticic, B. Baatar, J. Bartke, L. Betev, H. Białkowska, C. Blume, B. Boimska, J. Bracinik, V. Cerny, O. Chvala et al. (NA49 Collaboration), Eur. Phys. J. C 65, 9 (2010).

[47] A. Aduszkiewicz, Y. Ali, E. Andronov, T. Antićić, B. Baatar, M. Baszczyk, S. Bhosale, A. Blondel, M. Bogomilov, A. Brandin, A. Bravar et al., Eur. Phys. J. C 77, 671 (2017).

[48] R. Aaij et al. (LHCb Collaboration), Phys. Rev. Lett. 121, 222001 (2018).

[49] M. di Mauro, F. Donato, A. Goudelis, and P. D. Serpico, Phys. Rev. D 90, 085017 (2014).

[50] S.-J. Lin, X.-J. Bi, J. Feng, P.-F. Yin, and Z.-H. Yu, Phys. Rev. D 96, 123010 (2017).

[51] F. Donato, M. Korsmeier, and M. Di Mauro, Phys. Rev. D 96, 043007 (2017).

[52] M. W. Winkler, J. Cosmol. Astropart. Phys. 02 (2017) 048.

[53] M. Korsmeier, F. Donato, and M. Di Mauro, Phys. Rev. D 97, 103019 (2018).

[54] See Supplemental Material at http://link.aps.org/supplemental/ 10.1103/PhysRevResearch.2.023022 for more information about the technical points of the analysis.

[55] D. Maurin, Comput. Phys. Commun. 247, 106942 (2020).

[56] M. Korsmeier and A. Cuoco, Phys. Rev. D 94, 123019 (2016).

[57] J. Feng, N. Tomassetti, and A. Oliva, Phys. Rev. D 94, 123007 (2016).

[58] M. Kachelrieß, A. Neronov, and D. V. Semikoz, Phys. Rev. Lett. 115, 181103 (2015).

[59] Y. Génolini, M. Boudaud et al., Phys. Rev. D 99, 123028 (2019).
[60] L. Derome, D. Maurin, P. Salati, M. Boudaud, Y. Génolini, and P. Kunzé, Astron. Astrophys. 627, A158 (2019).

[61] F. James and M. Roos, Comput. Phys. Commun. 10, 343 (1975).

[62] G. Di Bernardo, C. Evoli, D. Gaggero, D. Grasso, and L. Maccione, Astropart. Phys. 34, 274 (2010).

[63] M. Aguilar, D. Aisa, B. Alpat, A. Alvino, G. Ambrosi, K. Andeen, L. Arruda, N. Attig, P. Azzarello, A. Bachlechner et al., Phys. Rev. Lett. 114, 171103 (2015).

[64] M. Aguilar, L. Ali Cavasonza, B. Alpat, G. Ambrosi, L. Arruda, N. Attig, S. Aupetit, P. Azzarello, A. Bachlechner, F. Barao et al., Phys. Rev. Lett. 119, 251101 (2017).

[65] K. Lodders, Ap. J. 591, 1220 (2003).

[66] J. J. Engelmann, P. Ferrando, A. Soutoul, P. Goret, and E. Juliusson, A\&A 233, 96 (1990).

[67] M. Garcia-Munoz, G. M. Mason, and J. A. Simpson, Ap. J. 202, 265 (1975)

[68] D. Casadei and V. Bindi, Ap. J. 612, 262 (2004).

[69] P. M. O’Neill, Adv. Space Res. 37, 1727 (2006).

[70] Y. Shikaze, S. Haino, K. Abe, H. Fuke, T. Hams, K. C. Kim, Y. Makida, S. Matsuda, J. W. Mitchell, A. A. Moiseev et al., Astropart. Phys. 28, 154 (2007).

[71] A. Ghelfi, F. Barao, L. Derome, and D. Maurin, A\&A 605, C2 (2017).

[72] M. S. Potgieter, Living Rev. Sol. Phys. 10, 3 (2013).

[73] J. S. Perko, A\&A 184, 119 (1987).

[74] A. Vittino, C. Evoli, and D. Gaggero, PoS ICRC2017, 024 (2018).

[75] M. Cirelli, D. Gaggero, G. Giesen, M. Taoso, and A. Urbano, J. Cosmol. Astropart. Phys. 12 (2014) 045.

[76] M. Aguilar, L. Ali Cavasonza, G. Ambrosi, L. Arruda, N. Attig, S. Aupetit, P. Azzarello, A. Bachlechner, F. Barao, A. Barrau et al., Phys. Rev. Lett. 120, 021101 (2018).

[77] R. Andrae, T. Schulze-Hartung, and P. Melchior, arXiv:1012.3754.

[78] D. Gaggero, A. Urbano, M. Valli, and P. Ullio, Phys. Rev. D 91, 083012 (2015).

[79] A. Putze, L. Derome, D. Maurin, L. Perotto, and R. Taillet, A\&A 497, 991 (2009).

[80] O. Adriani, Y. Akaike, K. Asano, Y. Asaoka, M. G. Bagliesi, E. Berti, G. Bigongiari, W. R. Binns, S. Bonechi, M. Bongi et al. (Calet Collaboration), Phys. Rev. Lett. 122, 181102 (2019). 